\title{
A Novel Real-Time Vehicle Tracking System Using Arduino
}

\author{
Abdullah H. Y. Alquhali, Mardeni Bin Roslee* \\ Centre for Wireless Technology, Faculty of Engineering, Multimedia University, MALAYSIA \\ *Corresponding Contact: \\ Email: mardeni.roslee@mmu.edu.my
}

\begin{abstract}
With the rapid increase in advancement in the world of technology, it has become highly important to the people to have the flexibility to monitor and control their physical property as well as personal data. Although physical transportation systems have made it easier for people to travel, it has also become a hazard in several ways. Therefore, a real-time tracking device has been designed in this project to cater to the issues of monitoring of vehicles. In this paper, we developed a system using Arduino Uno R3, Global System for Mobile (GSM) device and Global Positioning System (GPS) to track the exact and accurate position of the vehicle at a location. Arduino IDE software is utilized to code the Arduino Uno R3 and its microcontroller. The system is also equipped with display facilities to show the information to the user. The latitude and longitude of the vehicle's location are displayed on Liquid Crystal Display (LCD). Furthermore, two software is utilized to display the data. ThingSpeak was used to display the trend of the vehicle's motion by using charts of latitude and longitude, and Freeboard is utilized to display the same information in the form of a map. This map could easily be read by the user to pinpoint the exact location of the vehicle at any given time. It will be highly useful to monitor the exact locations of the delivery vehicle used by food-related businesses. Finally, by having latitude and longitude, users can successfully track their vehicle location on electronic maps using internet. Thus, the entire system has been tested thoroughly in real time and it is said to function successfully in helping users to locate their vehicles in the event of a theft.
\end{abstract}

Key words:

Global Positioning System (GPS), ThingSpeak, Global System for Mobile

\section{INTRODUCTION}

This paper presents the design of the Android based GPS system to track moving vehicles. This is an efficient tracking system built for the companies, authorities and public so that they can track their vehicles to avoid thefts. This section states the general background for 
choosing this specific project, the motivation for this project, the concept behind the design, the objectives of the project, the scope and also, the problem statement that prompted me to create this project. Previously, the technology that was being used in order to keep tracking on the vehicle was either the central dispatch system or a radio communication in the twoway method to continue the communication between the transmitter and the receiver. This was mainly used by several government departments, like police and fire trucks, and were also used by companies for delivery good, and taxi and car providing services. This twoway communication method was a dangerous hazard for the vehicles that were on the road, this was due to the driver, driving by one hand, while using the radio transmitter by another. This lead too many accident, due to less of focus on driving on the busy road. In order to reduce the number of these cases, we develop a smart vehicle tracking system which will help users to locate their vehicles in the event of a theft. This system is developed using Arduino Uno as a microprocessor, as well as GPS and GSM to coordinate geographical location of the stolen vehicle. By using these coordinates, users can track their vehicle position using Google Maps. To better assist the users in tracking down their vehicle, the information provided will be in real-time. The whole system is controlled by Arduino Uno. This Arduino is interfaced to LCD to display longitude and latitude. Apart from that, this system is also interfaced to GPS and GSM modem. The user can use the latitude and longitude provided to check the location in the Google Map. The user can access the location via internet by using Freeboard which is a dashboard for the Internet-of-Things application. The Freeboard will show the location of vehicle in Google Maps form and only authorized user can login into the Freeboard and share the link with people.

\section{Problem Statement}

The crime involving vehicle theft has been increasing in the past few years in Malaysia and from the latest update by General Insurance Association of Malaysia (PIAM) shows that about 60 vehicles were stolen every day and there are 11,796 cases from January until June 2016 and the numbers keep increasing until today. Any type of vehicle can be targeted by the thief and stolen vehicles are generally sold or in worst case scenario, some parts of vehicle might be separated and detached to be sold to different people, hence making it more difficult to find the vehicle. Before the vehicle tracking system was introduced, the users have to report to the police manually. The police have to initially investigate and then start to search for the car, while at the same time, the car might have already been moved far away from its original location. This problem, generally, is wasting time and is causing trouble to not only the user, but the police to find the car. Thus, knowing how important and crucial the vehicle tracking system for the safety purposes is, we are proposing this project. In this project the design and implementation of the vehicle tracking anti-theft system is proposed. This system not only secures and protects the moving vehicles, but also uses real time GPS system along with the GSM which will help the user in determining the precise location of the vehicle. This system will update the user for every instant of the moving vehicle through their GPS receiver.

\section{LITERATURE REVIEW}

There are some related works. Research done by Rahman et al. (2016) proposed an Arduinobased vehicle tracking system that utilized the technology of GPS to track the location of the vehicle, regarding of if it is in motion or is idle. It also utilized the technology of GSM to display the coordinates of the position of the vehicle on the cell phone using the Google map as a userfriendly application. The entire system processed in real-time. The system provides better 
service and cost effective solution for users. A vehicle's geographic coordinates obtained from an in-vehicle device. The system was able to experimentally demonstrate its effective performance to track vehicles location anytime from anywhere. This is easy to make and inexpensive compared to others. The project offered safety to the user as well as the vehicle itself. Further research has been done on a similar field where the proposed system allowed the distance travelled by the vehicle could be calculated and stored in the database for further analysis in future. The data could be viewed in real-time or at a later time. Arduino Uno was utilized to create this design and the incorporation of GPS and GSM allowed the system to transmit the coordinates of the position of the vehicle in real-time to the mobile phone of the user (Coifman et al., 1998). Further research has been done involving Internet of Things (IoT) (Abitha and Shyni, 2016) make the tracking device easier to communicate with other electronic devices. The paper focused on the design of a school bus monitoring system. A predictions algorithm was utilized to code the system such that the time of departure and arrival of the bus could be predicted using the route of the bus. The prototype also included an application that could be installed and launched on any smart phone. The limitation that this author project had was that the application did not allow the data to be stored so that the parents or the user could view the statistics later in future. This provided a limitation to the project as that information could be used to optimize the route that the bus takes. When the accuracy of the results were measured, it was concluded that they results were 100 percent accurate and the machine learning algorithm used to train and test the system able to predict the arrival time with high accuracy.

This system (Pachica et al., 2017) can be used in the monitoring our car, also in tracking the theft of the vehicle and in many more other applications. GPS is small tracking device that is connected to a car and the tracking software that is loaded from the software is used to provide feedback data. In this paper, a GPS based vehicle navigation system is implemented. This is done by utilizing GPS/GSM to convey the information of the vehicle such as location, distance travelled, etc. The time interval between the collections of data is specified by the user. Therefore, after every prefixed time duration the data is collected. Then this periodic data of the position of the vehicle is transmitted to tracking/ monitoring server. Additionally, to make the Global Positioning System (GPS) more accurate, the satellite will send the coordinates of longitude and latitude to the system to receive the critical data. This system utilizes microcontroller, Global System for Mobile Communication (GSM), and Global Positioning System (GPS) (Saad et al., 2018). This system uses only one GPS device that facilitates a two-way communication exchange. The functionality of the GSM modem is similar to the regular process of communication in a phone in terms of the requirement of a SIM card for both systems. Therefore, it can be concluded that the system requires an uninterrupted internet access to avoid any hindrance in the system. If the internet access discontinues for any reason, the communication will break off until the net is connected again. The proposed GPS/GSM based System contains two parts. The first part is the mobile unit and the second is a controlling station.

All system processes including the various interfaces for transmission of data have worked successfully. Then, these results are compatible with current GPS technologies (Brockman, 2017). A Vehicle Tracking System is a device that is fitted in a vehicle, to enable the vehicle owner to identify the vehicle's location. Based on the research done previously, this author paper proposes the design of GPS and GSM technology based vehicle tracking system. Utilizing the GPS and the GSM together, the system can track the vehicle and other objects based on an embedded system. Through this design, the location of the vehicle that is being used can be monitored regularly. Further, it will also report the status of the vehicle in 
demand. This paper (Dey et al., 2017) notes that remote signals can be transmitted through the GSM net-work. This includes two sectors: the monitoring center and the remote observation station. First, a computer and the GSM communication module comprises the monitoring center. VB was utilized for the software-monitoring center and the remote monitoring station. When the results of this project is analyzed, it can be demonstrated that the communication between the monitoring center and the remote monitoring station system can be controlled by the system. This proposed system (Mimar, 2016) uses cloud computing infrastructure as its basis. Sensors are utilized to supervise the driving settings including the velocity at which the car (or any other vehicle) is traveling, and even fuel level. The GSM unit then sends the information to the cloud server. Therefore, GPS antenna in installed in all the vehicles to identify locations. Similarly, (Zhang et al., 2005) an alcohol sensor can be attached to the vehicle to problems such as avoid drunk driving. These sensors would monitor the driver's alcohol status. The technology proposed in this author paper can therefore drastically reduce frequency of accidents on highways.

\section{MetHodology}

In this project, the major purpose is to track distinct vehicle that are traveling on the roads. The vehicle being tracked can transfer the information of the receiver such as location and it's speed, etc. This device is installed inside the vehicle. The tracking system on a moving vehicle basically works on the basis of the satellite through which the signals are being transmitted and received. The block diagram of the GPS tracking system for anti-thefting is shown in Figure 1.

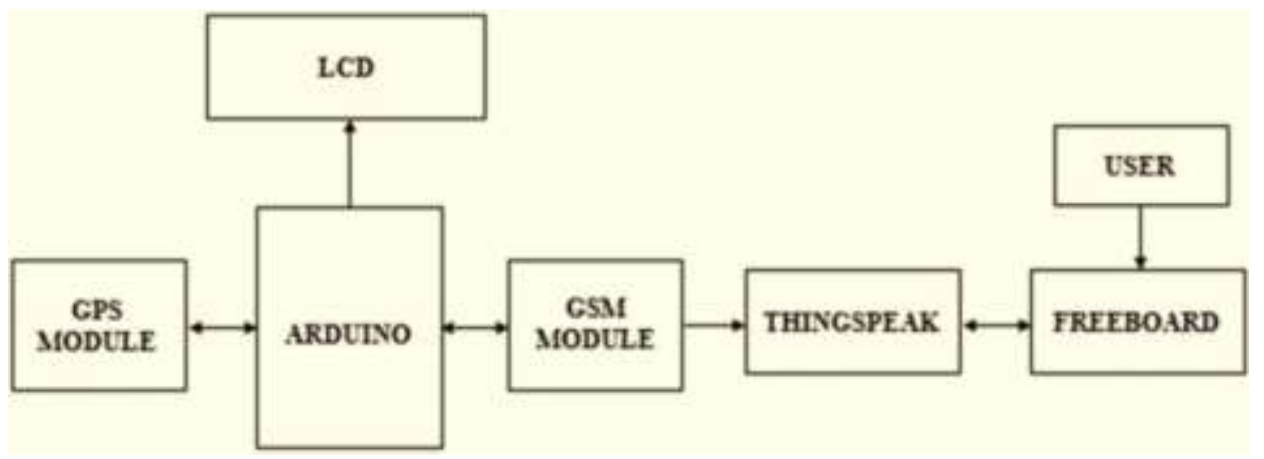

Figure 1: Block diagram of Real-time vehicle tracking using Arduino

This design in used to transmit the location and speed of the vehicle to the receiver's side. The most important part of this project is the transmission of the signals from the GSM module to the GPS receiver. An Arduino UNO has been used to manage the entire mechanism of the vehicle tracking system. Since a receiver and a transmitter is required for a tracking system, a GPS has been used to act as a receiver and a GSM module has been used to act as a transmitter. The GPS will identify the coordinates of the location at which the vehicle is at, while the GSM module will transmit those coordinates to ThingSpeak via SIM card internet data. A16x2 LCD connected to Arduino UNO will display the detected coordinates and show status messages of the detected coordinates. ThingSpeak is utilized as the platform where it stores the coordinates that GSM module send to it and Freeboard as the platform for user to seek their vehicle location. In this project, we have used ThingSpeak, Free-board, GPS Module GYNEO6MV2 and GSM Module SIM900A as the hardware to form a complete system. Furthermore, in this project the major hardware used as following: 


\section{Arduino Uno R3}

The microcontroller used to design this project is Arduino Uno R3. It has been chosen over other types of Arduino Uno products because it is the most advanced in terms of design and usage. The board has been integrated with the ATmega328 microcontroller (Kamisan et al., 2017).

\section{SIM900A GSM/GPRS Module}

SIM900A module is a complete Quad-Band GSM / GPRS module that incorporates the GPS mechanism with the satellite tracking system. The module comes with a feature called toolkit that allows a SIM card to be infused in it. The benefit of this module is that it is designed to be small in size and is therefore, very compact. Whereas the toolkit is small, it combines the properties of both GPS and GPRS in SMT package, and the features is containing is more. It is time-efficient and cost-efficient for developments that require such the use of both (Song et al., 2018). Additionally, the module comes up with an easy connection facility where it can be attached via USB ports. Therefore, no extra cables or interface is required to connect it with a GSP network. The GSM module is used to communicate to the system. In this work, a SIM900A hardware functioned in this project is to transfer the data from Arduino to ThingSpeak via sim card internet data.

\section{GY-NEO6MV2 GPS Module}

The GY-NEO6MV2 is a complete GPS engine module that features super sensitivity, ultralow power and small form factor. This board contains features such as a built-in EEPROM for processing, an antenna for communication and the u-blox NEO-6M GPS. These features make the board compatible with several controller boards that require the usage of a GPS module in their applications. Its $-161 \mathrm{dBm}$ tracking sensitivity extends positioning accuracy with PPP+SBAS and must be normal in a situation with unhampered view of the sky amid a certain period of time. In this project, this hardware (GPS module) is used to continuous collect the coordinates of vehicle. This paper discusses the process of developing a Vehicle Tracking Device (VTD). VTD is a tracking device focusing on vehicles that use Short Message Service (SMS). VTD will give information of location coordinate to mobile phone whenever there is a request for it through the SMS. The integrated Global Positioning System and Global System for Mobile communication (GPS-GSM) is used to track vehicle using application such as Waze or Google Maps (Hannan et al., 2015). This research work explores how to avoid this kind of stealing and provides more security to the vehicles.

\section{Liquid Crystal Display}

A liquid-crystal display (LCD) is a device that is used to display data or messages as per the application of the project. It consists of a simple flat panel that displays data utilizing external light properties. This is known as the light-modulating properties of liquid crystals. This means that the liquid crystals do not produce light on their own to display. Rather, they rely on the external light. In this project, the LCD displays the exact location of the vehicle via the longitude and the latitude of the earth using the tracking information given by GPS module

\section{Software Components}

In this work, a tracking device for a vehicle has been built which can be imbedded directly into a car, truck or any other vehicle and can be utilized to monitor the route and location 
of the vehicle in real time. During the tracking process, the GPS receiver would receive the information in the form of longitude and latitude of the position of the vehicle as read by the satellite. The information is then sent via GSM device to ThingSpeak and simultaneously is displayed on the LCD screen. Both GPS and GSM devices are connected via Arduino Uno R3 microcontroller. The geographical location along with the geographical coordinates are then displayed on the map using the Freeboard software which is an HTML-based platform. Since the coordinates of the vehicle's location are updated every 5 seconds, ThingSpeak assists in creating a database which can be accessed later at any time. The JavaScript of the browser allows the information to be displayed through simple charts to show the trend on the browser page and it also integrates the trend into the maps for the user.

\section{ThingSpeak}

ThingSpeak is an Internet of Things analytics platform service that allows you to aggregate, visualize and analyze live data streams in the cloud (Maureira et al., 2016). ThingSpeak provides instant visualizations of data posted by your devices to ThingSpeak. With the ability to execute MATLAB code in ThingSpeak, it can be performed online analysis and processing of the data as it comes in. ThingSpeak shows the longitude and latitude at which the vehicle is situated at. ThingSpeak shows the data that have been collected by the GPS module where it has been update to ThingSpeak via GSM module vis SIM card internet data. Thus, the ThingSpeak will show the same value that have been show in LCD.

\section{Freeboard}

Freeboard is an HTML-based platform that allows data to be displayed using usercustomized layouts. The user can add features to the display, which is usually displayed on a browser page, according to their own application necessities. The framework of Freeboard allows it to fetch information from the sources of the data. Thereby, collecting data and representing it in a simple way to the user using the widgets. The main positive aspect of the platform is that it gives flexibility to the user to avoid the setting up of a server for the processing of the data. Thus, reducing the overall cost of the data monitoring. Additionally, it gives convenience to the integrated devices to provide a presentable view of the complex designs of the webpage designs. The location of vehicle that can be track in real time using Freeboard. Freeboard is utilized to show the present position of the vehicle where the information has been collected from ThingSpeak then have been applied in this platform to show the latitude and longitude of vehicle in real time. Freeboard will be used as user platform to check their vehicle location.

\section{Arduino IDE}

To achieve a functional tracking system for the vehicle, the selection of a proper programming language was required that would be compatible with all the three devices, namely, Arduino Uno R3, GSM device and GPS device. Therefore, C-programming language has been utilized to code the Arduino Uno using Arduino IDE software. The coding utilizes void setup () to initiate the program whereas, void loop () executes the code. The same software was used to allow a successful transfer of data to and from the satellite. This allowed for a reliable transmission of data into the database via Arduino Uno R3. Arduino IDE software is an open source software which can be utilized to create a program according to the application required. The software compiles the code and uploads it into the micro controller on Arduino Uno's board. 


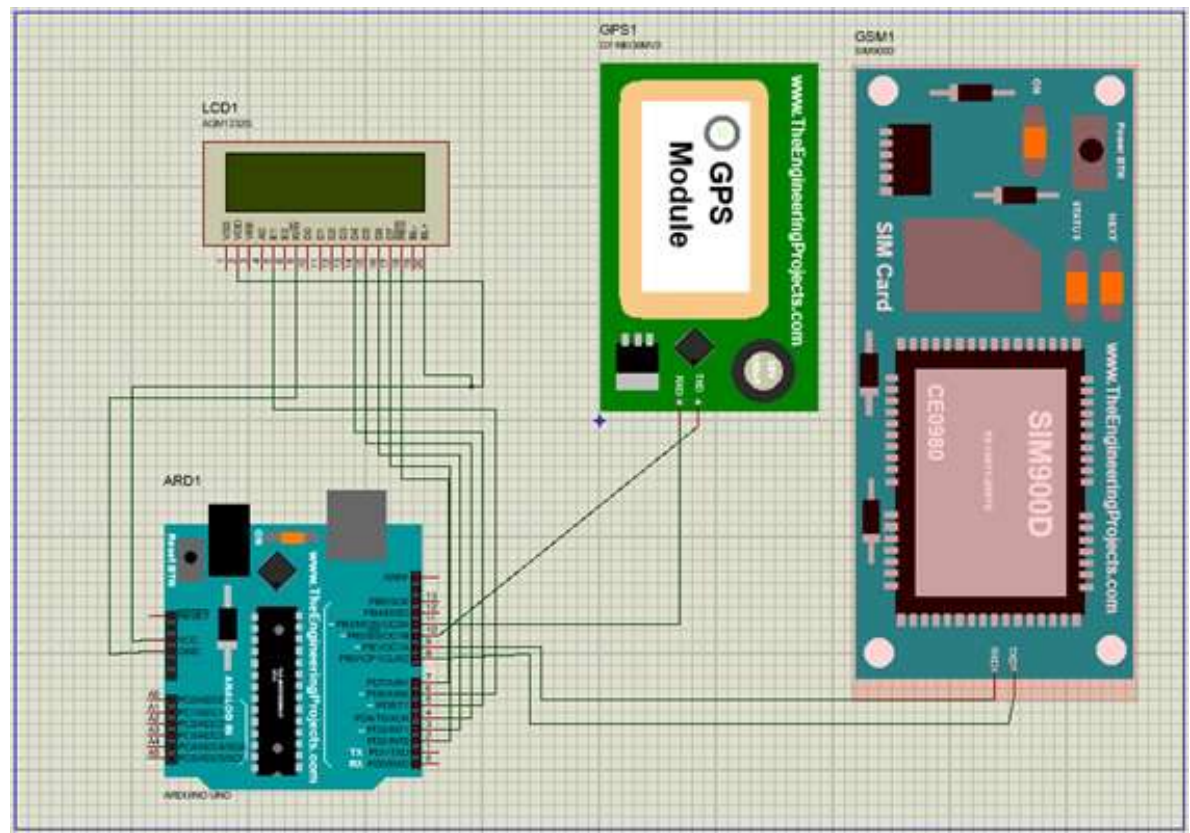

Figure 2: Circuit explanation

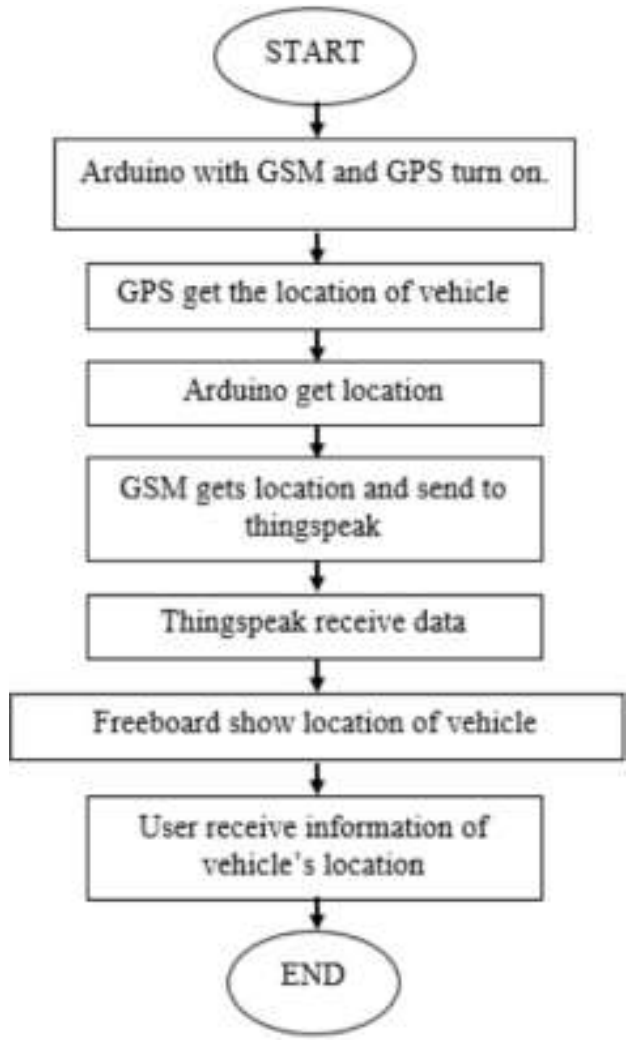

Figure 3: Flow Chart of Real-time Vehicle Tracking 


\section{RESULT AND Discussion}

This section presents the results and discussion of vehicle tracking system, where the circuit is made from several components which comprise of Arduino UNO board, GY-NEO6MV2 GPS module, SIM900A GSM module, rechargeable battery, 2x16 crystal liquid display (LCD) and the wire connection. The proposed vehicle tracking system. Figure 4 show the complete circuit of real-time vehicle tracking for theft prevention using Arduino inside the PVC box which is hiding in a boot compartment of a car.

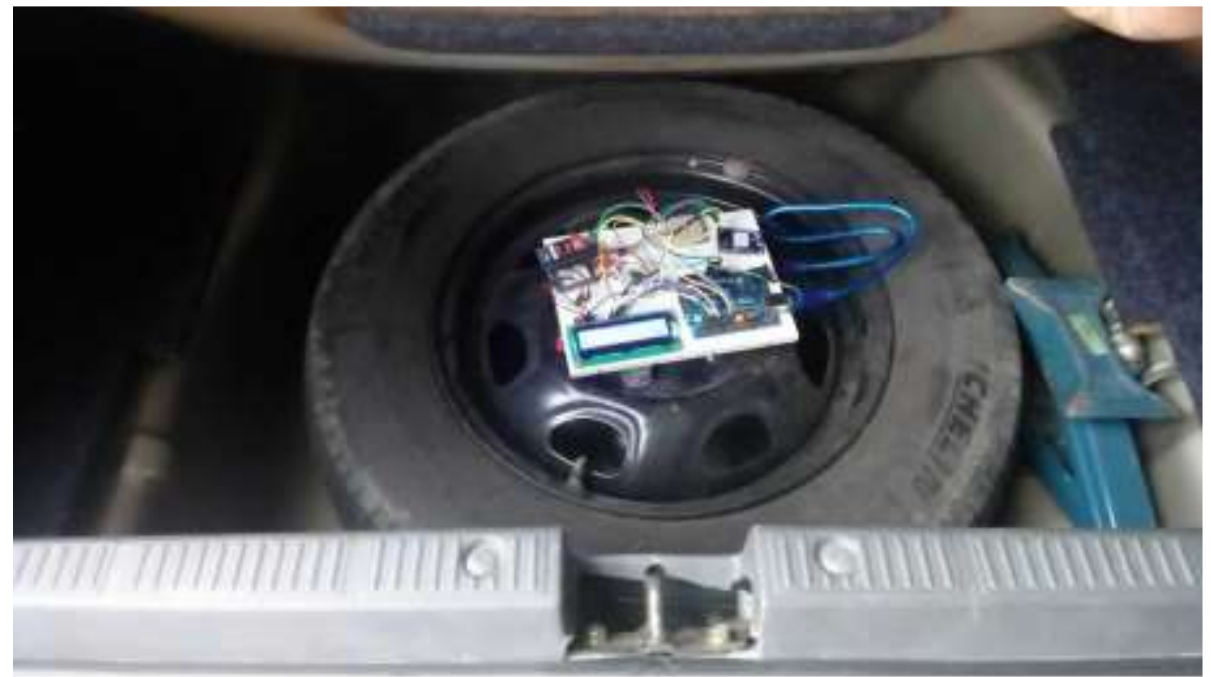

Figure 4: Complete circuit of Real-time vehicle tracking for theft prevention using Arduino

GPS module (GPS Module GY- NEO6MV2) will retrieve the location of the vehicle. The information retrieved will be sent using SIM800L module to ThingSpeak website and then the location is exported from Thingspeak website to be displayed in freeboard website. However, before uploading the values of latitude and longitude to the Thingspeak Arduino software must read the values from the GPS and show it in the Serial Monitor of the software as shown in Figure 5 The values that is going to be uploaded to the Thingspeak is same as the values that has been displayed in the serial monitor. The values are updated every 5 seconds. Therefore, if the vehicle is in motion, the values of latitude and longitude would change when GPS module will send the updated values.

\section{Results Display on Liquid Crystal Display}

Figure 5 shows that the exact latitude and longitude of the current vehicle's location is displayed on LCD after GSM module receive the information of vehicle location from Arduino. Since, the values of latitude and longitude are updated after every 5 seconds, the LCD display subsequently displays those values.

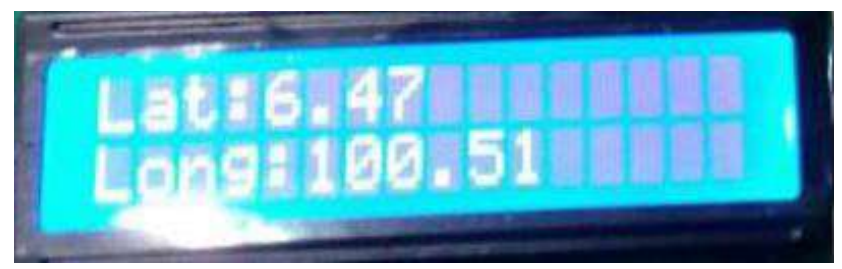

Figure 5: Current latitude and longitude of vehicle location display on LCD 


\section{Results Display on ThingSpeak}

Many experiments have been done to examine the robustness of the system by responding to the changes of the vehicle location in different places and updating the Thingspeak with the new values of the latitude and longitude in the new location. The vehicle was stopped at the first location and the results are shown in Figure 8 After the location of the vehicle was changed, the values of latitude and longitude changed on the LCD display, the Serial Monitor of Arduino software and the database maintained by ThingSpeak. The two results of experiments that have been done is discussed here refer to the Figure 6 shows one of the locations and Figure 7 and Figure 8 are shown the latitude and longitude values of the second location where it is been changed based on the new location of the vehicle. This shows the accuracy of the system by responding to any changes that could happened to the vehicle location.

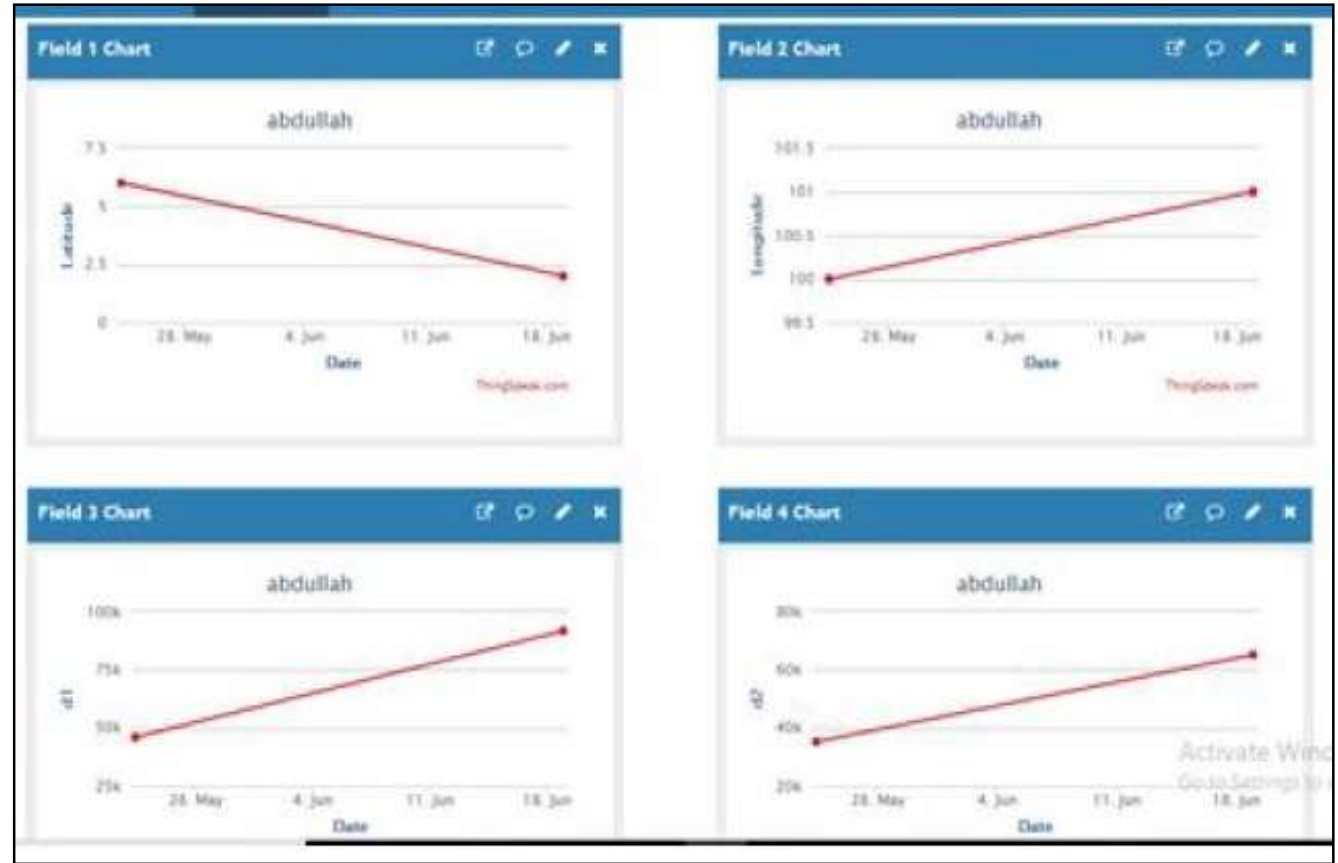

Figure 6: Current Values of Longitude and Latitude
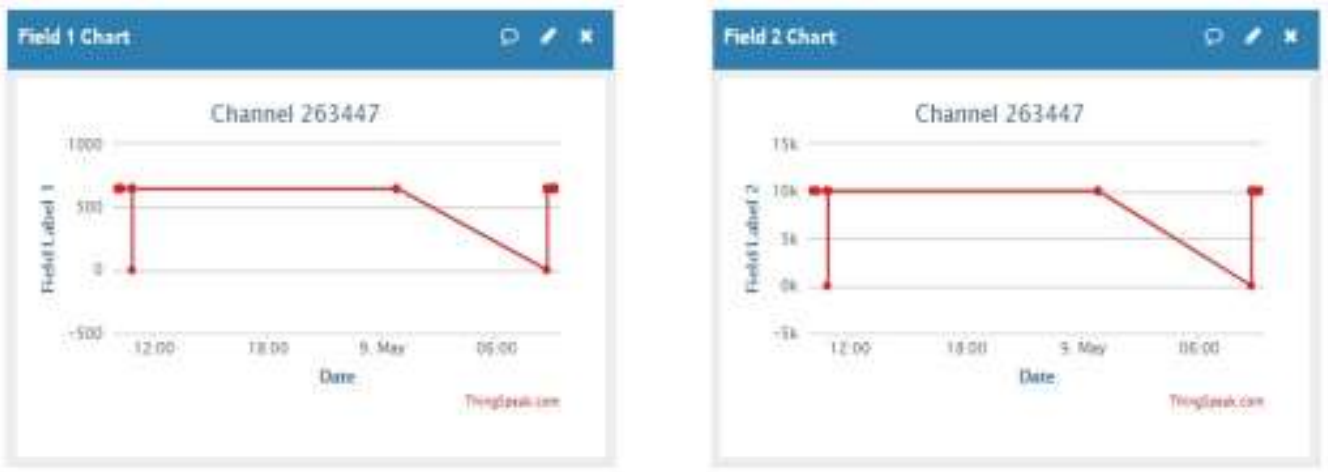

Figure 7: ThingSpeak received data by Arduino 


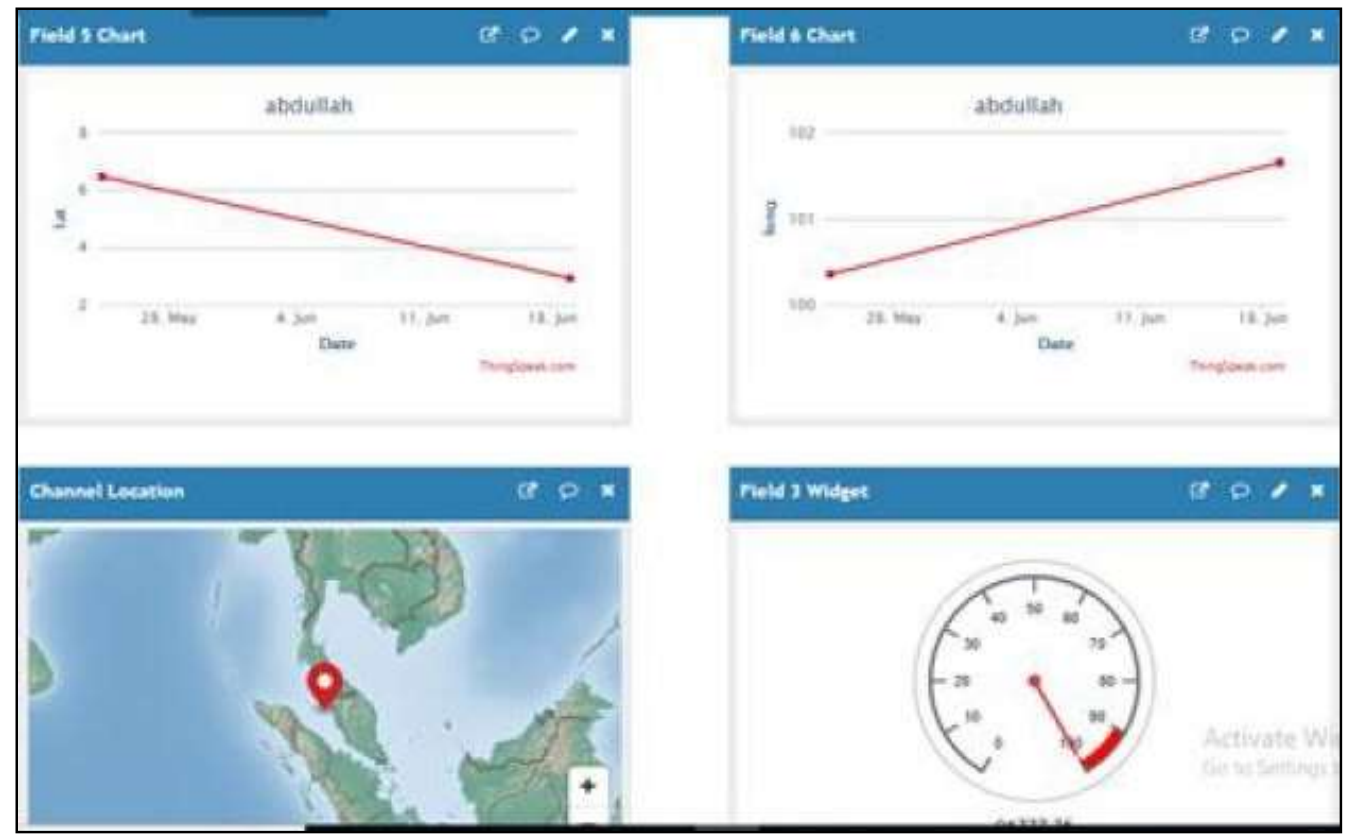

Figure 8: Example of vehicle at another location when ThingSpeak received data

\section{Result Display on Freeboard Software}

The following Figure 9 shows the location of vehicle is displayed on freeboard in the form of latitude, longitude and Google map. The latitude of the vehicle can be seen on the bottom left of the freeboard, while the longitude is the bottom in the middle. The date and time can also be seen as in the bottom right corner of page. At the same time, users can also see the exact location in the Google Map which is shown by the red marker in the Figure 10. The map can be zoomed in using the proper icon at the bottom right corner of the map to look at the exact location of the vehicle at that time.

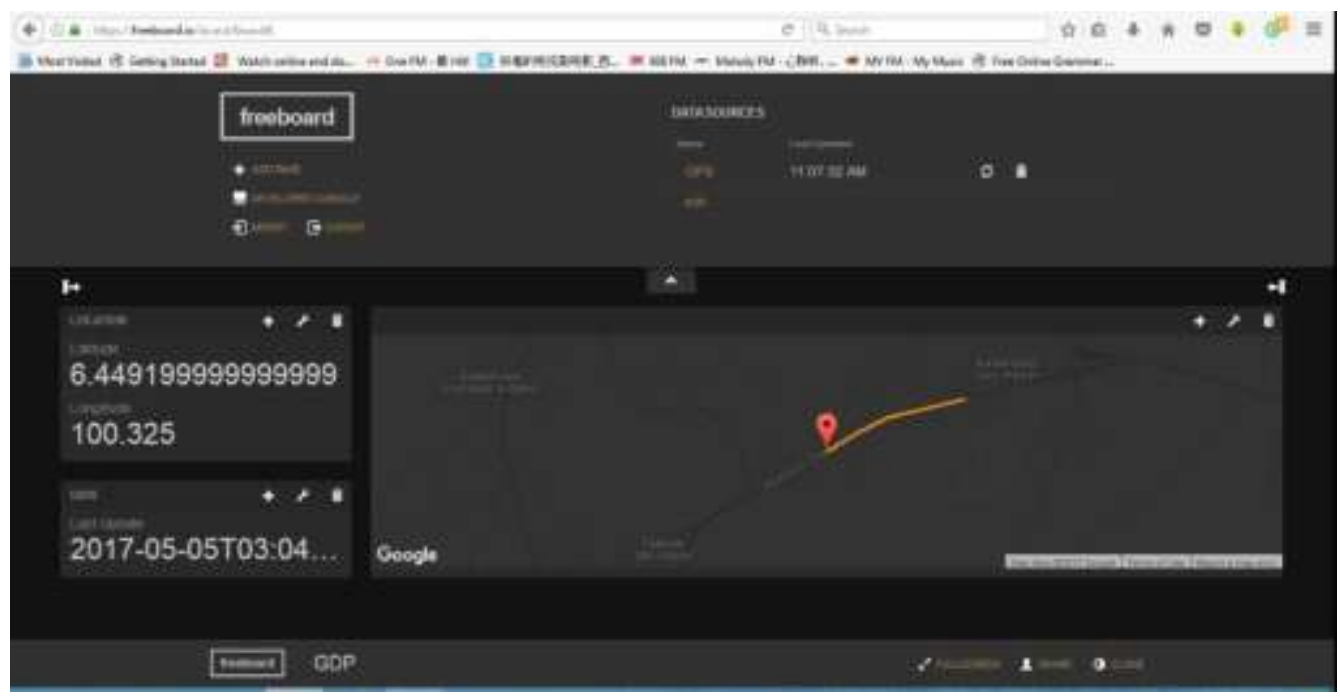

Figure 9: Vehicle location on Freeboard 


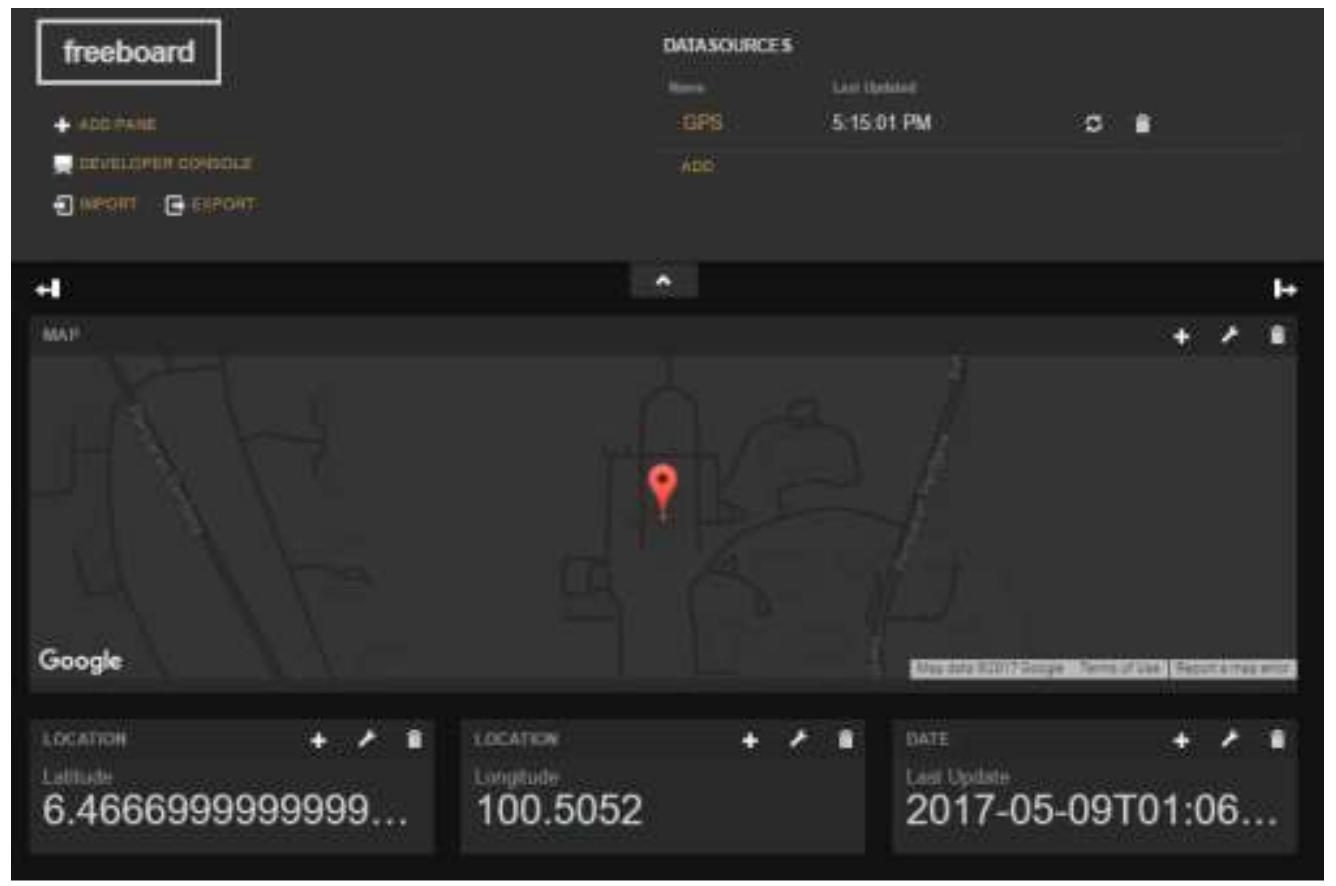

Figure 10: Vehicle at another location on Freeboard

A practical system for tracking vehicle is presented in this project. The system may be used in several applications. The system can be used by private owners of the vehicles track their vehicle if and when it is stolen or lost. It can also be used by owners of vehicle businesses such as taxi cab owners to track the location of all the vehicles in operation. The system can also be attached to school or company buses to allow the parents of the students or the employees respectively to monitor the track of the bus and to predict the departure and arrival time. Furthermore, it will be highly useful to monitor the exact locations of the delivery vehicle used by food-related businesses. The system starts when the user accesses into Freeboard account and then the system will show the details of the current location of vehicle in real time system. The details of longitude and latitude were taken from GPS in real-time. The user or police can use the latitude and longitude provided to check the location of the vehicle in the Google Map or use the link of freeboard that user share to police to find the lost vehicle in case of lost vehicle. The installation of the system in vehicle also must be done carefully to avoid any damage and trouble. The system must be put somewhere in hidden place around the vehicle so that the thief cannot find and ruin it easily. Thus, the system will be put in the locked box to avoid any problem.

As in the tracking system, this project involves GPS and GSM to track the vehicle and transmit the location of the vehicle. The GPS is utilized to provide the location of the vehicle in terms of the exact longitude and latitude of the geographical location, and the GSM is used to send the real-time location to ThingSpeak. If the place has no network, then the GPS will not function properly. This will make the location untraceable, hence causing difficulty to find the vehicle. ThingSpeak maintains a database of the data that it receives and represents it in a simple form of charts. The charts represent the stored values by showing trends of longitude and latitude. Freeboard displays the exact location of the vehicle using those values on a map, thereby making it user friendly to the purposes of analysis. 
The accuracy of the system was analyzed by changing the location of the vehicle and monitoring changes in latitude and longitude. As seen from the results in the previous section, this system was quite robust to variability of the location. The precision of the system was high as the GPS was able to update the location every 5 seconds. Since the precision depends on the position of the satellites in the orbit and the location of the vehicle read by GPS may vary if the position of the satellite changes while the GPS is sending the data, the precision was not 100 percent. The analysis of reliability of the system was done by experimenting it by placing it indoors and outdoors. When the GPS was placed outdoors in an open area, the GPS was able to connect to the nearby satellite at a faster rate and was able to transmit the data. However, the system became less reliable when the GPS was placed in an urban area. The time duration required by the GPS was relatively longer because the signals of the GPS bounced off the obstacles such as tall buildings. This caused the signals to be attenuated and the power of the signal reduced, but the data was successfully sent to ThingSpeak. So the performance of the system was high even in crowded places in busy cities. When the GPS was placed indoors, the GPS's reliability decreased drastically. Due to the low quality GPS board used for the purpose of the experimentation, the GPS was not able to connect to a nearby satellite in most cases.

\section{CONCLUSION}

In this work, the smart vehicle tracking system which is controlled by arduino Uno has been designed and tested successfully. In order to provide an efficient tracking system, the information provided need to be in real-time. It has been developed by integrating features of all the hardware components used. The Arduino is interfaced to GPS and GSM module. The GPS and smartphone used to coordinate geographical location of the stolen vehicle. The GSM module is used to send data via internet to the ThingSpeak.

Freeboard will receive the data from ThingSpeak and display the data in Google maps. User can see the movement of the vehicle in the Freeboard. By having latitude and longitude, users can track their vehicle location on electronic maps using internet. Thus, the entire system has been tested thoroughly in real time and it is said to function successfully in helping users to locate their vehicles in the event of a theft. The accuracy, precision and reliability of the system was analyzed by placing it in geographical locations with various accessibility of satellites. The accuracy and precision of the system was quite high and the accurate location of the vehicle was verified even when the vehicle was in a crowded place. However, the reliability of the system decreased gradually when the GPS was transported from an outdoor remote place to an outdoor urban area to an indoor area such as an indoor parking lot. The robustness of the system was measured by continuously and rapidly changing the position of the vehicle and by analyzing the results, it can be concluded that the system was highly robust in coping up with the variation of the latitude and longitude and was able to detect the exact position in a short time duration.

\section{REFERENCES}

Abitha, M., \& Shyni, S. (2016). AES algorithm for Smartphone to vehicle communication. 2016 International Conference on Computation of Power, Energy Information and Commuincation (ICCPEIC), 796-799.

Brockman, R.T. (2017). Vehicle tracking systems and methods, September 28. US Patent App. $15 / 618,567$.

Coifman, B.; Beymer, D.; McLauchlan, P; and Malik, J. (1998) A realtime computer vision system for vehicle tracking and traffic surveillance. Transportation Research Part C: Emerging Technologies, 6(4):271-288, 1998. 
Dey, M., Arif, M.A., \& Mahmud, M.A. (2017). Anti-theft protection of vehicle by GSM \& GPS with fingerprint verification. 2017 International Conference on Electrical, Computer and Communication Engineering (ECCE), 916-920.

Hannan, M.A., Al Mamun, M.A., Hussain, A., Basri, H., and Begum, R.A. (2015) A review on technologies and their usage in solid waste monitoring and management systems: Issues and challenges. Waste Management, 43:509-523.

Kamisan, M.T., Aziz, A.A., Ahmad, W.R., \& Khairudin, N. (2017). UiTM campus bus tracking system using Arduino based and smartphone application. 2017 IEEE 15th Student Conference on Research and Development (SCOReD), 137-141.

Maureira, MAG., Oldenhof, D., and Teernstra, L. (2016) Thingspeak-an api and web service. Leiden: [sn].

Mimar, T. (2016) Vehicle security with accident notification and embedded driver analytics, November 8. US Patent 9,491,420.

Pachica, A. O.; Barsalote, D. S.; Geraga, J. M. P.; Ong J. M.; and Sajulan, M. D. (2017). "Motorcycle theft prevention and recovery security system," 2017 14th International Conference on Electrical Engineering/Electronics, Computer, Telecommunications and Information Technology (ECTI-CON), Phuket, 2017, pp. 850-855. doi: 10.1109/ECTICon.2017.8096372

Rahman, M.M.; Mou, J.R.; Tara, K. and Sarkar, M.I. (2016) Real time google map and arduino based vehicle tracking system. In Electrical, Computer \& Telecommunication Engineering (ICECTE), International Conference on, pages 1-4. IEEE.

Saad, S.A., Hisham, A.A.B., Ishak, M.H., Fauzi, M.H., Baharudin, M.A., \& Idris, N.H. (2018). Real-time on-campus public transportation monitoring system. 2018 IEEE 14th International Colloquium on Signal Processing \& Its Applications (CSPA), 215-220.

Song, D., Tharmarasa, R., Kirubarajan, T., \& Fernando, X.N. (2018). Multi-Vehicle Tracking With Road Maps and Car-Following Models. IEEE Transactions on Intelligent Transportation Systems, 19, 13751386.

Zhang, P., Shi, Z., \& Xu, M. (2005). Design and implementation of vehicle monitoring system based on GPRS. 2005 International Conference on Machine Learning and Cybernetics, 6, 3574-3578 Vol. 6. 
DOI: https://doi.org/10.18034/ei.v6i2.228

Online Archive Link: https://abc.us.org/ojs/index.php/ei/issue/archive 